\title{
Fermented Medicinal Plant Designed by Lactobacillus Hits Alternative Complement Pathway and Controlled hA1c Level
}

\section{Yamaguchi $\mathbf{N}^{1,2 *}$, Akazawa-Kudoh $\mathrm{S}^{3}$, Sawada $\mathbf{M}^{3}$, Fujimoto $\mathrm{Y}^{3}$ and Murayama $\mathrm{T}^{4}$}

${ }^{1}$ Ishikawa Natural Medicinal Products Research Center, Ishikawa, Japan

${ }^{2}$ Department of Fundament Research for CAM, Kanazawa Medical University, Ishikawa, Japan

${ }^{3}$ Department of General Medicine, Kanazawa Medical University, Ishikawa, Japan

${ }^{4}$ Department of Microbiology and Immunology, Faculty of Pharmaceutical Sciences, Hokuriku University, Ishikawa, 920-1181, Japan

\begin{abstract}
Background: A Type II Diabetes mellitus showed many symptoms that brought serious for the patients. One of the problem was suppressed activity of the phagocyte for the foreign microorganism.
\end{abstract}

Objective: The objective of this study was to find excellent method to recover the suppressed phagocytic cell activity both in animal and human.

Methods: A wild medicinal herbs were prepared by Yeast and Lactobaccilli against 80 sorts of wild plants including medicinal remedies (FWP80). These products were exhibited by safe in animal safety test. The trial was made to investigate a recovery of immune-competent cells both in mice and human that exhibited phagocytic depression.

Results: Our results in animal model showed that FWP80 augmented the macrophage activity, both chemotaxis and phagocytosis. In clinical study with 20 volunteers diagnosed type II DM, the granulocyte and lymphocyte ratio was produced to as modulate as neutral, 30 days after the introduction by FWP80.

Conclusion: In mice, depressed phagocytic cell by Streptozotocin, the macrophage activities were recovered by FWP80. We proposed an idea that FWP80 exhibited remedy effect via controlling complement component. The mechanism of activation was proved by activating alternative pathway of complement by the technic, immuneelectrophoresis. We tried to evaluate more to the anti-oxidative activities of FWP80, directory employing peritoneal macrophage in vivo.

Keywords: Lactobacillus; Fermentation; Cancer chemotherapeutic agent; Compromised host; DM; Alternative complement activation

Abbreviations: CAM: Complementary and Alternative Medicine; CD: Cluster of Differentiation; DM: Diabetes mellitus; F80: Fermented wild herbs consisted with 80 sorts of herbs and fruits that had been depredated to micro fragment by Lactobacillus; FCM: Flow Cytometry; G-rich type: An individual that exhibit over $60 \%$ of granulocyte in peripheral blood, finding many in young gentleman; L-rich type: An individual that exhibit over $40 \%$ of lymphocyte in peripheral blood, finding lot in ladies and senile

\section{Introduction}

About defence system of vertebrate, the complexed problems of developing our dual system, the innate and inductive do not seem to guard or even protect the maturation of one internal threat to survival. Also, all the individual suffers from the risk of immunodeficiency in daily activities with both internal and/or externals events, such personal habitat of individual, such in lifestyle related disease [1-5]. Recently, complementary, and alternative medicines (CAM) have interested more and more attractive since they are able to treat many life-style related diseases, such as Diabetes mellitus, fatigue syndrome that spurred in the industrialized world. The present report had showed that typical styles of CAM, preparing special molecule for both digestive and easy to augmented human complement component that regulated the functions of leukocytes in human immune defence system [6-10]. We had been published and proposed that the health promoting supplement could work after depredated the polysaccharide to appropriate fragment they possible to activate complement component. This was especially being part of alternative complement pathway. Dietary Supplements and fermented derivatives suggested as potent agent food supplement for regulating an acquired immunity through alternative complement pathway. These processes were close concollection of the complement activating process different from invasion of infectious agent where the immune complex and/or toxic polysaccharide such as LPS worked as pathway of complement. On the other hand the immune system is working for the local infection of microorganisms, antigen presenting cell directed throughout the immune system referred to the endocrine and nervous system In this report, we hypothesized that FWP80 might acted for immuno-competent cells qualitatively and quantitatively, activating lymphocytes according to the constitution dependent manner. FWP80 had been employed as remedy and the implication has little been made on the characteristics of the levels of leukocyte subset, such as granulocytes and lymphocytes ratio. In this paper, we also proposed to focus on the identification of FWP80 formula, relating to another herbal medicine that was the first line of CAM. The influence of FWP80 on its ratio to leukocyte and/or lymphocyte subpopulations was also discussed. The overall mechanism of FWP80 to the phagocytic cell is also discussed in concern to the complement activation especially alternative complement pathway. This phenomenon was only find to prepare chained saccharide that enable to activate the complement pathway, made by acidophilic bacteria and yeast fungus [10].

\section{Methods}

\section{Preparation of Conventional 80 and Fermented 80}

Commercially available wild herbal remedies were processed and

*Corresponding author: Nobuo Yamaguchi, Ishikawa Natural Medicinal Products Research Center, Ishikawa, Japan, Tel: +817622258852; E-mail: serumaya@kanazawa-med.ac.jp

Received March 21, 2017; Accepted March 31, 2017; Published April 05, 2017

Citation: Yamaguchi N, Akazawa-Kudoh S, Sawada M, Fujimoto Y, Murayama T (2017) Fermented Medicinal Plant Designed by Lactobacillus Hits Alternative Complement Pathway and Controlled hA1c Level. Nat Prod Chem Res 5: 260. doi: 10.4172/2329-6836.1000260

Copyright: (c) 2017 Yamaguchi N, et al. This is an open-access article distributed under the terms of the Creative Commons Attribution License, which permits unrestricted use, distribution, and reproduction in any medium, provided the original author and source are credited. 
extracted by $100 \mathrm{ml}$ of hot water $\left(98^{\circ} \mathrm{C}\right)$ to 10 gr of grained and extracted for 30 minutes. The micronization was carried by Lactobacillus leuteria for 6 months at $30^{\circ} \mathrm{C}$, and was repeated two times in the method described in $\mathrm{M} \& \mathrm{M}$. After the centrifugation of $2000 \mathrm{xg}$ for $10 \mathrm{~min}$ at room temperature and then, dried by heating as powder and served for FWP80.

\section{Fermentation and GABA generation}

Commercially available wild herbal remedies, Manyoh were purchased by Echigoyakusoh, Co. Ltd. Niigata, Japan. The fermentation was prepared by Lactobacillus leuteria for 6 months at $20^{\circ} \mathrm{C}$. GABA: gamma amino acid butyric acid was traced by the check system $[9,10]$.

In order to prove the fragmentation of the material, FWP80, we employed simple method to probe the positive materials that fermented by Lactobacilli instead of HPLC: high pressure liquid chromatograph. GABA: $\gamma$-aminobutyric acid detecting. Was employed by the probe of fermentation and degradation. GABA, reduced nicotinamide adenine di-nucleotide phosphate by using a specific enzyme, aminotransferase and a dehydrogenase which correspond to the steps of producing reduced nicotinamide adenine di-nucleotide phosphate employing a specific enzyme, aminotransferase and a dehydrogenase that works to make oxidized nicotinamide adenine di-nucleotide phosphate as a coenzyme and inactivating the enzymes, therefore inactivating any amino acid exhibiting a same structure to that of GABA and acting an electron carrier on NADPH produced in the following step in the mixture of a tetrazolium salt that can produce a water-soluble formazan dye and estimating the water-soluble formazan dye evidenced and reported in references $[11,12]$.

\section{Animal experiment}

Ten female seven-week-old ddY mice, were employed for the acute oral toxicity study. The check systems were carried out according to Ethics of the Organization for Economic Co-operation and Development (OECD) Test Guideline 401. The mice were kept at $24 \pm$ $1^{\circ} \mathrm{C}, 50 \%$ relative humidity.

\section{Experimentally induced model for diabetes mellitus by Streptzotosin as immune-suppressed mice}

For the animal model of immune suppression, male C57BL/6J mice, aged 8-9 weeks, were injected with Streptozotocin $(5 \mathrm{mg} / \mathrm{kg})$ to induce diabetes mellitus in mice. In order to established immune -defected as well as DW mice, C\%'BL/6 mice were employed, injecting $5 \mathrm{mg} / \mathrm{kg}$ of Streptozotocin.

At a dosage, $1 \mathrm{~g} / \mathrm{kg}$ of FWP80 were administered to the immune suppressed Black mice for 7 days. Then, FWP80 derivatives was introduced orally at a dosage of $1 \mathrm{~g} / \mathrm{kg} /$ day for seven consecutive days. Conventional FWP 80 was selected as controls of the experiment. One week later, their blood was withdrawn from their tail vain. Then, the number of leukocytes was checked in Bürker-Türk solution.

\section{Recovery of phagocytic cell activity, migration}

A cilled RPMI-1640 medium were injected mice peritoneal and collected $80 \%$ of original volume from the cavity after massaging of the mice peritoneal.

A glass adherent cell was prepared by the method reported before and $1 \times 10^{4}$ cell were put into the upper room of Boyden Chamber. Nucleated cells from peritoneal fluid were collected from the peritoneal cavity of immune-suppressed mice. Glass adherent cells were purified using glass adherent technique to purify the cell suspensions which gathered more than $95 \%$ of phagocytes. The purified cells were set on to the upper part of Boyden chamber to evaluate migration activity at a concentration of $1 \times 10^{4} \mathrm{cell} / \mathrm{ml}$. and Human serum treated at $56^{\circ} \mathrm{C}$ for $30 \mathrm{~min}$ was for the chemo tactic factor of mouse phagocyte. [1,10,13] 90 minutes after the incubation, Millipore membrane was washed by warmed RPMI-1640 and then fixed by formalin. Then the membrane was stained by May-Giemsa solution and recorded by photometry and counted.

\section{Activation of phagocytic activity}

A chilled RPMI-1640 medium were injected mice peritoneal and collected $80 \%$ of original volume from the cavity after massaging of the mice peritoneal.

A glass adherent cell was prepared by the method reported before and $1 \times 10^{4}$ cell were put into the upper room of Boyden Chamber. The same cells preparation was purified by glass adherent method for macrophage purification, which prepare the cells contained more than $95 \%$ of phagocytes. The purified cells were prepared into $1 \times 10^{4} \mathrm{cell} /$ $\mathrm{cm}^{2}$ and mixed with latex beads that are adjusted in $5 \mu \mathrm{m}$ of diameter and conjugated with fluorescence Isocyanate. After incubation with 90 minutes, remained granules were washed out three times from the glass slide. Number of macrophage and their ability to catch up the latex beads were automatically measured by ACAS system, which drove the result in a digital form (Adherent cell activity evaluating system; Shimazu, Kyoto, Japan).

\section{Human Trial}

\section{Participants}

Thity-four participants were selected in this study (age $63 \pm 7.8$ ). They were given self-questionnaire on their blood sugar and hAlc levels prior to investigation. After noting their medical history, we selected individuals who would participate in the experiment voluntarily. All participants gave their written informed consent prior to participation. The two group were selected as randomized manner.

\section{Statistical analysis}

Data were analyzed employing the 2002-2003 SAS Version 9.1 software (SAS Institute Inc., Cary, NC, USA) represented as means \pm standard deviations. The differences between FWP80-treated and nontreated control were compared using a one-tailed analysis of variance. A $P$ value $<0.05$ was regarded to be statistically significant.

\section{Results}

\section{Animal safety test for FWP80}

Recovery of macrophage activity, chemotaxis by FWP80: Figure 1 showed that the group Streptozotocin clearly suppressed the chemotactic activity of mice both in number and function (Figure 1, middle). After administration of FWP80, the mice recovered their chemotactic activity to more than normal range. With a precise observation, the recovery activity was different in dose dependent manner, the strongest FWP80 among the three dosages as to augmentation in number and function of phagocytes (Figure 1, right). On the other hand, the augmentation by conventional one was less than that by fermented one, FWP80 (Figure 1).

Recovery of macrophage activity, phagocytosis by FWP80: After preparing the same number of phagocytic cells in the same stage of glass slide, Figure 2 showed that Streptozotocin clearly deceased number of phagocytic cell in the area, the macrophage phagocytic activity of 
Citation: Yamaguchi N, Akazawa-Kudoh S, Sawada M, Fujimoto Y, Murayama T (2017) Fermented Medicinal Plant Designed by Lactobacillus Hits Alternative Complement Pathway and Controlled hA1c Level. Nat Prod Chem Res 5: 260. doi: 10.4172/2329-6836.1000260

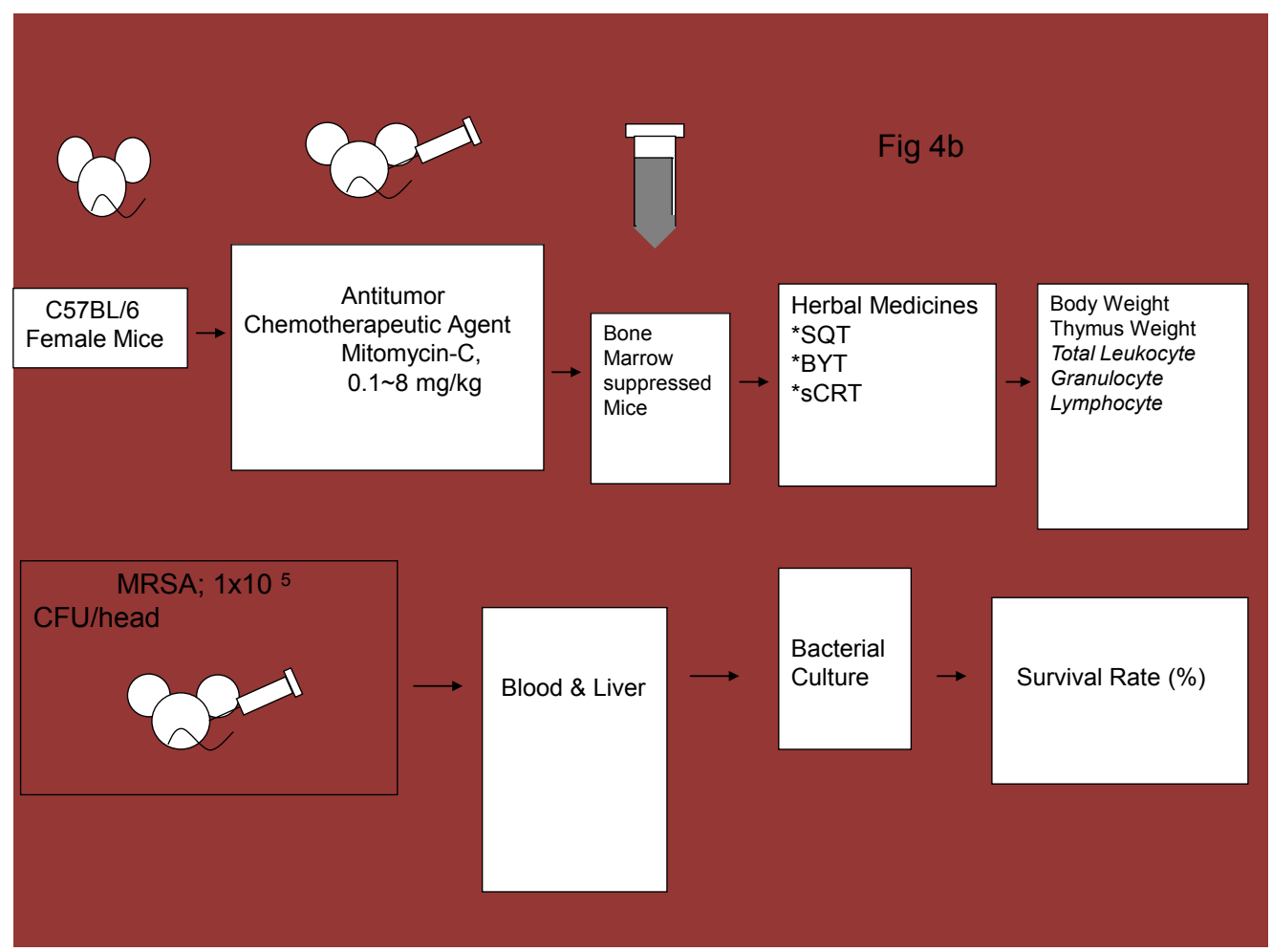

Figure 1: Experimental design for this report.

mice depressed both in number and function as indicated digitally to the amount of fluorescent labelled latex beads inside the cell (Figure 2, middle). After the treatment of FWP80, the mice recovered their phagocytic activity over normal range, showing rebound phenomenon. With a precise observation, the recovery activity was the strongest FWP80 among the three dose ranges as to augmentation in number and function of phagocytes (Figure 2, right) (Figure 2).

\section{Clinical Findings}

\section{Changes in cell number of total leukocyte and leukocyte subsets by FWP80}

Seventeen participants were finally selected in this study (age $63 \pm$ 7.8). They were all healthy volunteers to make informed and written consent of this trial. Leukocyte numbers have been counted one hour before and 30 days after the treatment of conventional or FWP80. We recorded the cell number that were obtained bone hour before starting the trial was $100 \%$. After 30 day of trial, the cell number were counted again and expressed in number and relative $\%$ in the Table 1.

\section{Dividing subjects into two groups, G-type and L-type according to granulocyte and lymphocyte proportion}

The volunteer were healthy normal 20 individuals. A total number of leukocyte was not significantly change after the administration of FWP80. On the other hand, the relative ration of leucocyte subset, granulocyte and lymphocyte were changed significantly before and after the trial (10). To further characterization to the influence of FWP80, we separated the subjects into two groups: the G type group, who had a granulocyte count over $60 \%$, and the L type group, who had a lymphocyte count over $40 \%$. In the L type group, lymphocyte counts derived to down-regulated on day 30 , accompanied by an increase in

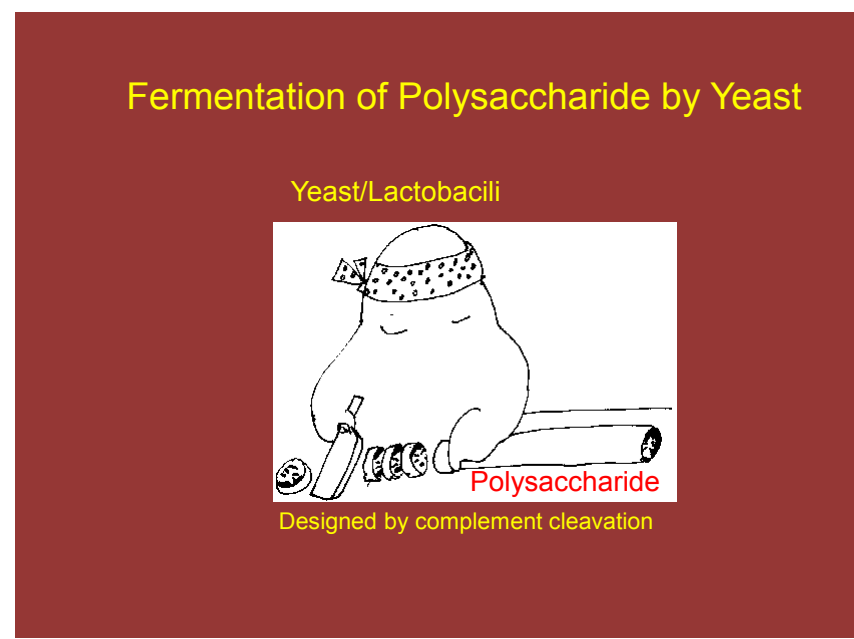

Figure 2: Illustrative imaging for degradation of polysaccharide by microorganism.

\begin{tabular}{|l|c|c|c|c|}
\hline \multirow{2}{*}{} & \multicolumn{2}{|c|}{ G type individual } & \multicolumn{2}{c|}{ L type individual } \\
\cline { 2 - 5 } & \multicolumn{2}{|c|}{$\mathbf{f - . 8 0}$} & \multicolumn{2}{c|}{$\mathbf{f - 8 0}$} \\
\cline { 2 - 5 } & Before & After & Before & After \\
\hline Total WBC $\left(\times 10^{3} \mu \mathrm{l}\right)$ & 6.44 & 5.78 & 3.47 & 5.65 \\
\hline Lymphocyte (\%) & 25.6 & 26.8 & 43.6 & 38.7 \\
\hline Granulocyte (\%) & 67.5 & 68.5 & 54.8 & 57.5 \\
\hline Neutrophil (\%) & 65.5 & 64.3 & 44.6 & 53.1 \\
\hline Eosinophil (\%) & 1.5 & 2.9 & 2.5 & 4.2 \\
\hline Basophil (\%) & 0.5 & 0.6 & 0.8 & 0.8 \\
\hline
\end{tabular}

Table 1: Constitution dependent regulation of leukocyte by F80. 
granulocyte numbers compared to control, conventional 80 but not by FWP80. On the other hand, the granulocyte counts of G type group indicated to decrease on day 30 . The decrease of granulocyte count was augmented by fermented FWP80, but not by conventional 80 on day 30 (10) (Table 2).

\section{Follow up study for haemoglobin hA1c level by FWP80}

Seventeen participants were finally selected in this study (age $63 \pm 7.8$ ). They were all healthy volunteers to make informed and written consent of this trial. Before starting FWP80 administration, haemoglobin hAlc level were followed up to 3 month. The results were shown in Table 3. The levels were stable at least up to 3 month, even the FWP80 was stock by sweet remedy from beat radish that contain partly with tandem mono-sugar molecule. (Table 3).

\section{The complement system-another step for exhibition of effect by fragmented polysaccharide, FWP80}

We would like to check on another important factor of defence component factor, Complement. These proteins are composed by at least 9 components. These proteins are famous for its protective activity against infectious bacteria in the defence immunity. However, we had found that the complement had worked when we introduced fragmented /fermented polysaccharide, FWP80, as complement activator, so called alternative pathway developed to Alternative Medicine. In this section, we would like to show the 1) what was the starting material in FWP80, 2) which pathway of complement did kick out for activation, 3) what kind of physical activity was augmented as a result of this process. So,

\begin{tabular}{|c|c|c|c|c|}
\hline \multirow{2}{*}{ CD } & \multicolumn{2}{|c|}{ G type individual } & \multicolumn{2}{c|}{ L type individual } \\
\cline { 2 - 5 } & \multicolumn{2}{|c|}{$\mathbf{f - 8 0}$} & \multicolumn{2}{c|}{$\mathbf{f - 8 0}$} \\
\cline { 2 - 5 } & Before (\%) & After (\%) & Before (\%) & After (\%) \\
\hline CD2 & 6.66 & 76.76 & 60.43 & 77.65 \\
\hline CD4 & 19.54 & 28.44 & 31.43 & 45.67 \\
\hline CD8 & 37.65 & 42.57 & 26.38 & 28.63 \\
\hline CD11 & 73.77 & 72.68 & 63.45 & 69.54 \\
\hline CD14 & 0.03 & 0.06 & 0.06 & 0.07 \\
\hline CD16 & 67.65 & 58.55 & 54.24 & 46.67 \\
\hline CD19 & 8.45 & 8.21 & 8.41 & 7.95 \\
\hline CD56 & 1.57 & 1.88 & 1.78 & 2.87 \\
\hline
\end{tabular}

Table 2: Constitution dependent regulation of lymphocyte by $F 80$.

\begin{tabular}{|c|c|c|c|c|}
\hline Volunteer No & Before & 1 Month & 2 Month & 3 Month \\
\hline 1 & 6.5 & 6.4 & 6.4 & 6.3 \\
\hline 2 & 8.1 & 8.0 & 7.8 & 7.8 \\
\hline 3 & 6.3 & 5.6 & 5.8 & 6.1 \\
\hline 4 & 5.8 & 5.5 & 5.6 & 5.5 \\
\hline 5 & 7.2 & 7.3 & 7.4 & 7.7 \\
\hline 6 & 7.5 & 7.6 & 7.4 & 7.2 \\
\hline 7 & 5.6 & 5.4 & 5.3 & 5.3 \\
\hline 8 & 6.8 & 6.8 & 6.3 & 6.2 \\
\hline 9 & 5.9 & 5.8 & 5.6 & 5.5 \\
\hline 10 & 5.9 & 5.4 & 5.5 & 5.3 \\
\hline 11 & 6.5 & 6.3 & 6.5 & 6.3 \\
\hline 12 & 6.4 & 6.5 & 6.3 & 6.3 \\
\hline 13 & 7.6 & 7.5 & 7.3 & 7.2 \\
\hline 14 & 7.3 & 6.9 & 6.7 & 6.6 \\
\hline 15 & 6.5 & 6.4 & 5.3 & 5.3 \\
\hline 16 & 7.2 & 7.1 & 6.9 & 6.5 \\
\hline 17 & 7.0 & 6.8 & 6.7 & 6.5 \\
\hline HA1c (Mean) & 6.33 & 6.55 & 6.4 & 6.33 \\
\hline & & & & \\
\hline
\end{tabular}

Table 3: Follow up study for hemoglobin A1c level by F80. in this report we would like to exhibit the character of complement and activated mechanism that lead to the augmentation of all the physical events through the activation of complement receptor positive structure cells. After generating complement component, various biological phenomenon was followed by activation, such as capillary swelling and scarlet phenomenon. These documented events were so called allergic processes. Activation of alternative of complement system results in a cascade of relation of these proteins, leading to the generation of products that have important biological functions such as swelling and scarlet phenomenon and that constitute an important humoral activating system involved in inflammatory site of the peripheral. We expected that the fragmented polysaccharide other than mono sugars, gently hit this alternative processes, leading to the total augmentation of physical activities. First, involvement of particles, such as bacteria or immune complexes, with certain parts of complement facilitates the digestion of the particle by macrophage etc., phagocytic cells (opsonic function of complement). Second, the activation event resulted many fission products of complement proteins for which specific receptors expressed on a variety of inflammatory cells, such as macrophage, neutrophil, lymphocytes, and other cells. Binding of these complement-derived elements to such receptors results in biologic activities such as chemotaxis and hormone-like activation of cellular activities $[10,13,14]$.

\section{The specific pattern of complement cascade and complement proteins by FWP80}

The two-major process were known to drive to make MAC: membrane attack complex. The process was known to classical pathway and alternative one. The complement elements could move by two separate processes: the classical and the alternative pathways. Both pathways guide to a common terminal pathfinder referred to as the pathway of membrane attack complex. Both the classical and the alternative complement pathways can be cascaded into various component units: initiation, amplification, and membrane attack complex. The initial recognition group, which leads to start of the complement processes, an integrative step result to that involves the activation of relevant enzyme and the reuse of additional elements. These are followed by an end phase of membrane attack complex: MAC that the target cell dies and next to autophagy phenomenon. The recognition unit for the classical pathway, the first component $\mathrm{C} 1$, is consisted of three independent components, $\mathrm{Clq}, \mathrm{Clr}$, and $\mathrm{Cls}$ which derived from complement recognition unit. The initiation of this pathfinder of complement typically drive the reaction of antibody plus antigen, which may be conjugated or on the surface of a target cell. Thus, the initiation of complement activation is started by the binding of antigen-antibody complex in the classical pathway of complement cascade. This antigen-antibody complex results the binding of $\mathrm{Clq}$ to two or more Fc regions of certain IgG subclasses (IgG1, IgG2, IgG,) or IgM. Activators of the classical pathway. The visualization employing higher magnification technique bring us to recognize to consist of six subunits similar to a component of six pedals relevant to the component of Clq. The multiple component of $\mathrm{Clq}$ has been visualized by electron microscopy to consist of six subunits similar to a component of six pedals. The other hit pathway, polysaccharide molecule, such as FWP80 also activated the complement component, especially alternative pathway. Then, some polysaccharide residue hit the complement element in the manner of alternative pathway. Thus, FWP80 element hit human complement element and indicated by immune electrophoretic visual precipitated methods. In our lymphoid fluid, the gentle processing of component of $\mathrm{Clq}$ are occur instead vigorous movement of infection by the microorganisms. 
Citation: Yamaguchi N, Akazawa-Kudoh S, Sawada M, Fujimoto Y, Murayama T (2017) Fermented Medicinal Plant Designed by Lactobacillus Hits Alternative Complement Pathway and Controlled hA1c Level. Nat Prod Chem Res 5: 260. doi: 10.4172/2329-6836.1000260

Page 5 of 6

Thus, naturally occurs consecutively in plasma at a low rate. The main structure of C3, C3a keep an initial structure, after thioester bond has been hydrolysed The $\mathrm{C} 3$ molecules in which the thioester bond has been hydrolyzed just like C3b, but the C3a domain has not been diapered. C3 with a hydrolysed thioester is named C3 or C3b-like C3. It can conjugate Factor B and allow Factor D then cleave to Factor B, which develop in construction of a fluid-phase $\mathrm{C} 3$ convertase, $\mathrm{C} 3, \mathrm{Bb}$. This enzyme is consecutively interacting and compose $\mathrm{c} 3 \mathrm{~b}$ molecules that can randomly attract to cells. Therefore, these C3b molecules will be promptly changed by the target cells by Factors $\mathrm{H}$ and I, the complement component will initiate the positive feedback construct on target surfaces, as indicated previously. On the other side, the alternative pathway is constantly activated at a low concentration of the starting material such as FWP80, but amplification with subsequent cell death induced only on foreign particles such recently known as autophagy [10,13-15].

In this regards, we tried to prove visually by the immuneelectrophoresis as immune-precipitation method. The human serum was collected 3 hours after oral administrating FWP80. Incubation is made to make precipitin line for $90 \mathrm{~min}$ and the dialyzed by physiological saline and then desolation and dried and coloured y blue dye for visualization. Immuno-electrophoresis was prepared for $90 \mathrm{~min}$, followed by incubating with anti-human whole serum and specific for C3 and Bf element. The specific anti human complement component antibodies were kindly presented by s by Dr Syunnosuke SAKAI, Cancer Research Institute of Kanazawa University, Japan [10] (Figures 3-5).
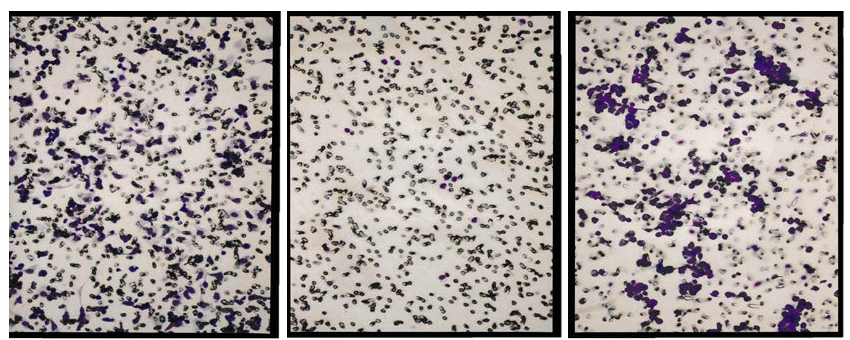

Figure 3: Macrophage chemotaxis judged by boyden chamber.

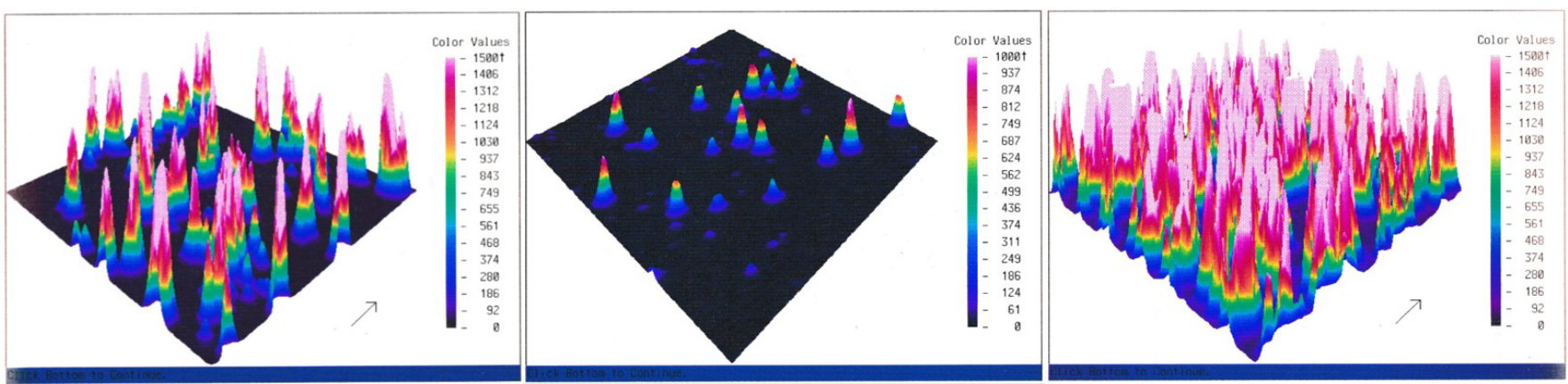

Figure 4: Macrophage phagocytosis against fluorescent activated latex beads.

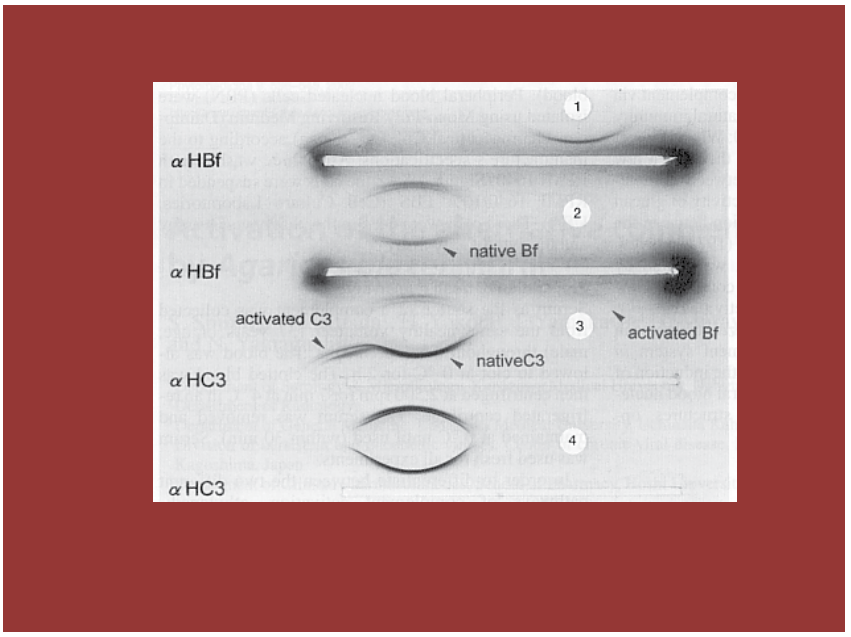

Figure 5: Immuno-electrophoretic demonstration of activated human complement 
Citation: Yamaguchi N, Akazawa-Kudoh S, Sawada M, Fujimoto Y, Murayama T (2017) Fermented Medicinal Plant Designed by Lactobacillus Hits Alternative Complement Pathway and Controlled hA1c Level. Nat Prod Chem Res 5: 260. doi: 10.4172/2329-6836.1000260

\section{Conclusion}

In rodents, compromised host by Streptozotocin, the macrophage activities, chemotaxis and phagocytosis were recovered by FWP80. We proposed a concept that FWP80 exhibited tonic effects via activating complement components such that though alternative complement pathway. With those effects by FWP80, the blood sugar level, hA1c controlled by long term administration by human volunteer.

\section{Conflict of Interest}

We declared that there was no conflict of interest in this study.

\section{Acknowledgements}

Declared none.

\section{References}

1. Suzanne EG, Andy IMH (1999) Immune dysfunction in patients with diabetes mellitus (DM). 26: 259-265.

2. Yamaguchi N, Takahashi T, Sugita T, Ichikawa K, Sakaihara S, et al. (2007) Acupuncture Regulates Leukocyte Subpopulations in Human Peripheral Blood. Evid Based Complement Alternat Med 4: 447-453.

3. Suzuki S, Toyabe S, Moroda T, Tada T, Tsukahara A, et al. (1997) Circadian Rhythm of Leukocytes and Lymphocytes Subsets and Its Possible Correlation with the Function of the Autonomic Nervous System. Clinical Experimental Immunology 110: 500-508.

4. Yamaguchi N, Kawada N, Ja XS, Okamoto K, Okuzumi K, et al. (2013) Overal Estimation of Anti-Oxidant Activity by Mammal Mammal Macrophage. OJRA 4: 13-21.

5. Hamada M, Yamaguchi N (1988) Effect of Kanpo Medicine, Zyuzentaihoto, on the immune reactivity of tumor-bearing mice. J Ethnopharmac 24: 311-320.
6. Yamaguchi N, Shimizu S, Izumi H (2004) Hydrotherapy Can Modulate Peripheral Leukocytes: An Approach to Alternative Medicine. Complementary and Alternative Approaches to Biomedicine 239-251.

7. Murayama T, Yamaguchi N, Matsuno H, Eizuru Y (2004) In Vitro AntiCytomegalovirus Activity of Kampo (Japanese Herbal) Medicine. eCAM 1: 285-289.

8. Abe S, Yamaguchi N, Tansho S, Yamaguchi H (2005) Preventive Effects of Juzen-taiho-to on Infectious Disease, Juzen-taiho-to (Shi-Quan-Da-Bu-Tang) Scientific Evaluation and Clinical Applications. In: Traditional Herbal Medicines for Modern Times. Yamada H, Ikuo Saiki I (eds.).

9. Yamaguchi N, Ueyama T, Amat N, Yimit D, Hoxur P et al. (2015) Bi-Directional Regulation by Chinese Herbal Formulae to Host and Parasite for Multi-Drug Resistant Staphylococcus aureus in Humans and Rodents. Open Journal of Immunology 5: 18-32.

10. Yamaguchi N, Araai M, Murayama T (2015) Aspect of QOL Assessment and Proposed New Scale for Evaluation. Open Journal of Immunology 5:47-182.

11. Wacharaporn N, Melike B, Tugba K, Filiz O, I'smail T (2012) Contribution of Gamma amino butyric acid (GABA) to salt stress responses of Nicotiana sylvestris CMSII mutant and wild type plants. Journal of Plant Physiology 169 : 452-458.

12. Thomas B, Boris O, Caol B (2012) Gamma-aminobutyric acid and glutamic acid level sintheauditory pathway of rats with chronic tinnitus: a direct determination using high resolution point-resolved proton magnetic resonance spectroscopy (1H-MRS). Frontier Systems Neuroscience 6: 1-12.

13. Jyumonji N, Fujii Y (1993) A new assay for delayed-type hypersensitivity in vitro Detection by the macrophage migration by Boyden chamber. J Kanazawa Med Univ 18: 198-203.

14. Fujita T, Wakamiya N, Ternor JA (2016) Preface to the special Issue for XXVI International Complement Workshop. Immunobiol 221: 1035-1036.

15. Sim RB, Shwable W, Fujita T (2016) Complement Research with 18th-21st Centuries: Progress come with new technology. Immunobiol 221: 1037-1045. 